\title{
Survival versus collapse: Abrupt drop of excitability kills the traveling pulse, while gradual change results in adaptation
}

\section{$\operatorname{AUTHOR}(S)$ :}

Tanaka, Masanobu; Nagahara, Hiroki; Kitahata, Hiroyuki; Krinsky, Valentin; Agladze, Konstantin I.; Yoshikawa, Kenichi

\section{CITATION:}

Tanaka, Masanobu ...[et al]. Survival versus collapse: Abrupt drop of excitability kills the traveling pulse, while gradual change results in adaptation. Physical Review E 2007, 76(1): 016205.

\section{ISSUE DATE:}

2007-07-10

URL:

http://hdl.handle.net/2433/45692

RIGHT:

Copyright (2007) by the American Physical Society. 


\title{
Survival versus collapse: Abrupt drop of excitability kills the traveling pulse, while gradual change results in adaptation
}

\author{
Masanobu Tanaka, Hiroki Nagahara, Hiroyuki Kitahata, Valentin Krinsky, Konstantin Agladze, and Kenichi Yoshikawa* \\ Department of Physics, Graduate School of Science, Kyoto University, Kyoto 606-8502, Japan \\ and Spatio-Temporal Project, ICORP JST, Kyoto 606-8502, Japan \\ (Received 21 February 2007; published 10 July 2007)
}

\begin{abstract}
Excitable media show changes in their basic characteristics that reflect temporal changes in the environment. In the photosensitive Belousov-Zhabotinsky (BZ) reaction, excitability is decreased by illumination. We found that a traveling pulse failed to propagate when a certain level of light intensity was switched on abruptly, but the pulse continued propagating when the light intensity reached the same level gradually. We investigated the mechanism of adaptation of pulse propagation to the change in light intensity using two mathematical models, the Oregonator model (a specific model for the photosensitive BZ reaction), and the FitzHugh-Nagumo model (a generic model for excitable media). The appearance of a characteristic such as adaptation is shown to be a general feature for a traveling pulse in excitable media.
\end{abstract}

DOI: 10.1103/PhysRevE.76.016205

PACS number(s): 05.45.-a, 82.40.Ck

\section{INTRODUCTION}

Excitability plays an important role in various systems in nature, such as heart tissues [1], nerve axons [2], the retina [3], aggregating amoebae [4], the catalytic $\mathrm{CO}$ oxidation reaction on a Pt surface [5], and the Belousov-Zhabotinsky (BZ) reaction $[6,7]$. These systems change their features depending on changes in environmental factors: temperature, brightness, pressure, etc.

The eye adjusts to changes in light. This process is called "adaptation." The human eye can work under very low to very high levels of light intensity-its sensing capabilities span nine orders of magnitude. Most of this adaptation to changes in light occurs in the retina. Under adaptation, the retina primes more or fewer photoreceptor molecules to receive light depending on the ambient brightness. In addition to sight, it is well known that adaptation is a characteristic of sensation in general [8]. Usually, adaptation is considered to be managed through a feedback mechanism via a neural circuit. However, "adaptation" may also involve some other mechanism, i.e., the excitability of terminal neurons can be modulated through time-dependent input from the external environment. In this study, we examined the latter possibility through an experimental system exhibiting excitability.

We examined the adaptation of a single excitation pulse to a temporal change in the environment by using the photosensitive BZ reaction $[9,10]$. Previously, Agladze and coworkers found that the development of a single rotating spiral wave depends on the rate of change in excitability [11]. In this chemical system, light illumination results in the production of bromide [12], which inhibits the chemical reaction. Thus, excitability can be easily controlled by changing the intensity of illumination as an environmental parameter $[13,14]$. We found experimentally that the nature of pulse propagation depends on the rate of change in light intensity. The experimental results can be reproduced well by a nu-

\footnotetext{
*Corresponding author. FAX: +81 75753 3779. Email address: yoshikaw@scphys.kyoto-u.ac.jp
}

merical simulation based on the Oregonator model $[15,16]$. Furthermore, we will interpret the essential features of adaptation by using the FitzHugh-Nagumo (FHN) model [17,18], which includes a generic mathematical structure in an excitable system.

\section{EXPERIMENTAL PROCEDURE}

We used the photosensitive $\mathrm{BZ}$ reaction with ruthenium $(\mathrm{Ru})$ catalyst as an experimental system. The final concentration of the photosensitive $\mathrm{BZ}$ solution was $\left[\mathrm{NaBrO}_{3}\right]$ $=0.6 \mathrm{M}, \quad\left[\mathrm{H}_{2} \mathrm{SO}_{4}\right]=0.3 \mathrm{M},\left[\mathrm{CH}_{2}(\mathrm{COOH})_{2}\right]=0.2 \mathrm{M}, \quad[\mathrm{NaBr}]$ $=0.05 M$, and $\left[\mathrm{Ru}(\mathrm{bpy})_{3} \mathrm{Cl}_{2}\right]=1.7 \mathrm{~m} M$, which is similar to that in the previous study [19]. Here bpy means bipyridyl $\left(\mathrm{C}_{10} \mathrm{~N}_{2} \mathrm{H}_{8}\right)$. A cellulose nitrate membrane filter (Advantec, A100A025A) with a pore size of $1.0 \mu \mathrm{m}$ was soaked in the BZ solution $(5 \mathrm{ml})$ for about $3 \mathrm{~min}$. The soaked filter was gently wiped with paper to remove excess solution and placed on a glass plate. The surface of the membrane filter was completely covered with about $1 \mathrm{ml}$ of silicone oil (Wako, WF-30) to avoid drying and to protect it from the influence of oxygen. The experiments were carried out in an air-conditioned room at $22 \pm 1{ }^{\circ} \mathrm{C}$. We confirmed that no spontaneous excitation was generated on the reaction field even without illumination.

The medium was illuminated from below, as shown schematically in Fig. 1. A liquid-crystal projector (Epson, ELP810) was used as a light source, and the spatiotemporal distribution of the light intensity was controlled by a personal computer. The nature of pulse propagation was monitored from above with a digital video camera (Panasonic, NVGS100) and recorded on videotape. For image enhancement, a blue optical filter (Asahi Techno Glass, V-42) with a maximum transparency at $410 \mathrm{~nm}$ was used. The light intensity at the illuminated area was measured with a light-intensity meter (As One, LX-100).

\section{EXPERIMENTAL RESULTS}

Figure 2(a) shows snapshots of pulses propagating on quasi-one-dimensional 1.0-mm-wide tracks (R1 and R2), 


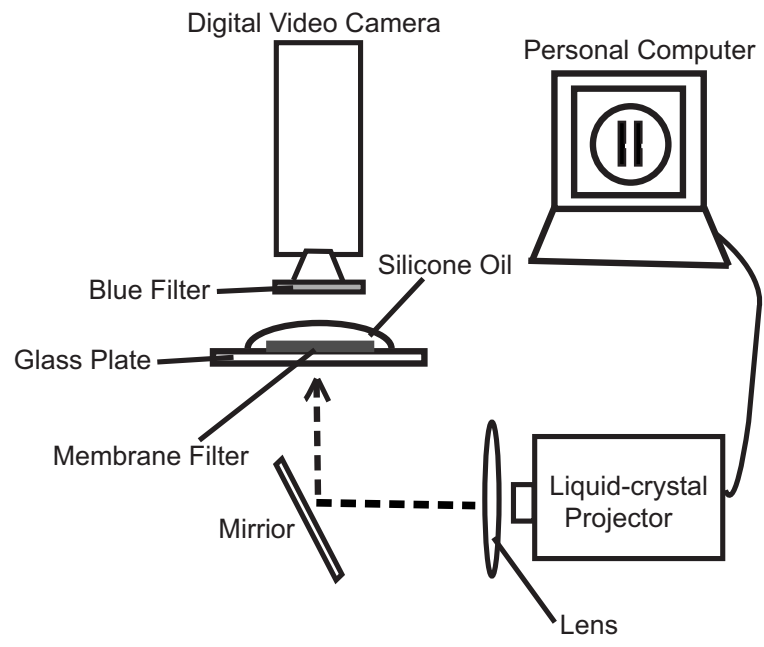

FIG. 1. Schematic illustration of the experimental system for the photosensitive BZ reaction.

where the tracks were illuminated at $0.5 \mathrm{klx}$ for $t<0$. At $t$ $=0$ the light intensity started to increase from 0.5 to $17 \mathrm{klx}$. Outside the tracks the field was illuminated continuously at $66 \mathrm{klx}$, which completely inhibited excitability. To investigate the effect of the rate of increase in light intensity on the nature of propagation of the excitation pulse, we changed light intensity from 0.5 to $17 \mathrm{klx}$ for tracks in both R1 and $\mathrm{R} 2$, where the duration of the change was 110 and $0.1 \mathrm{~s}$ in
R1 and R2, respectively. Figure 2(b) shows the experimental results for the photosensitive $\mathrm{BZ}$ reaction, presented as spatiotemporal plots of single-pulse propagation along R1 and R2, respectively. In Fig. 2(b), each bright line corresponds to the propagation of an excitation pulse. Under a slow change in light intensity, the single pulse propagated throughout the track to the farthest end (R1). On the other hand, under an abrupt change, the single pulse disappeared (R2).

Taken together, these results show that a pulse could not propagate when the light intensity was changed abruptly from 0.5 to $17 \mathrm{klx}$, while it was found that a stable excitation pulse can propagate under stationary conditions at both light intensities ( 0.5 and $17 \mathrm{klx})$. This can be translated as follows. A traveling pulse can be easily generated under lower light intensity; in this case, a pulse could not be generated by an abrupt increase in light intensity from an initial state with lower light intensity, but it could by a slow increase.

\section{NUMERICAL CALCULATION}

We have adopted the so-called two-variable Oregonator model to interpret the nature of pulse propagation in the photosensitive BZ medium $[15,16]$. To investigate the mechanism that underlies the observed phenomena, we performed numerical calculations using the modified Oregonator model $[20,21]$ for the photosensitive $\mathrm{BZ}$ reaction,

$$
\frac{\partial u}{\partial t}=D_{u} \nabla^{2} u+\frac{1}{\epsilon_{u}} f(u, v, \phi)
$$

(a)

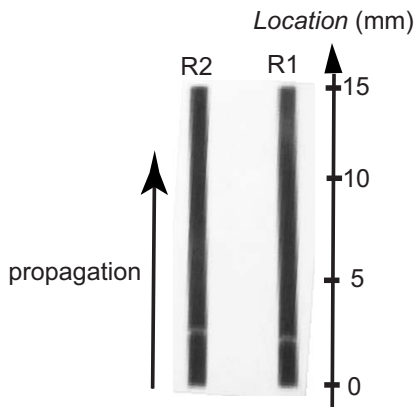

(b)

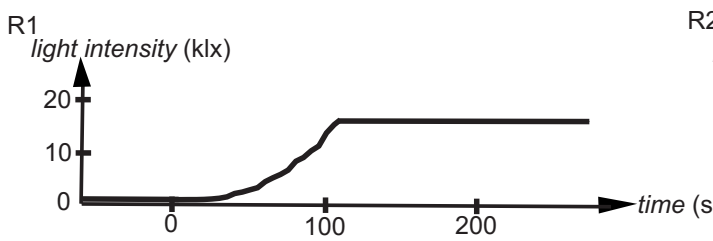

R2
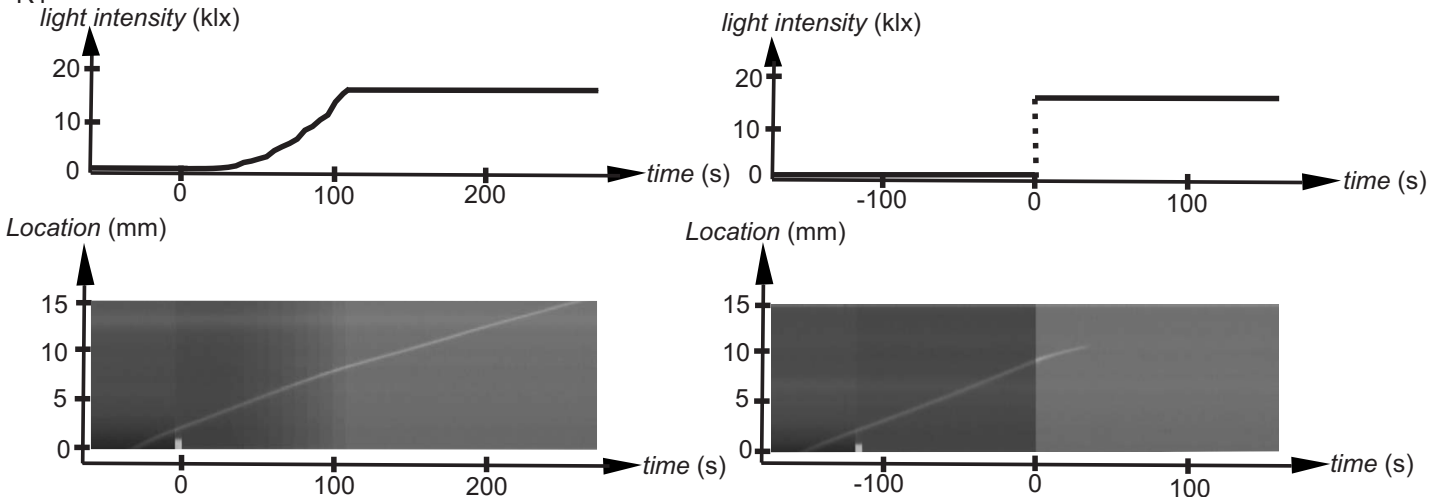

FIG. 2. Experimental results. (a) Top view of the reaction tracks at $t=0$ on $\mathrm{R} 1$ ( $t=-110$ on $\mathrm{R} 2)$. The white region was illuminated at $66 \mathrm{klx}$, and showed complete inhibition. The two stripes R1 and R2 were initially affected by a slight suppression effect at $0.5 \mathrm{klx}$. At $t$ $=0$ the light intensity started to increase to $17 \mathrm{klx}$ (largely suppressed state), and the rates of change were different between R1 and R2. (b) Spatiotemporal plots showing the different nature of pulse propagation between the tracks. The pulse propagated throughout the track under a slow change (R1), whereas it failed to propagate under an abrupt change in light intensity (R2). 
(a) (i)
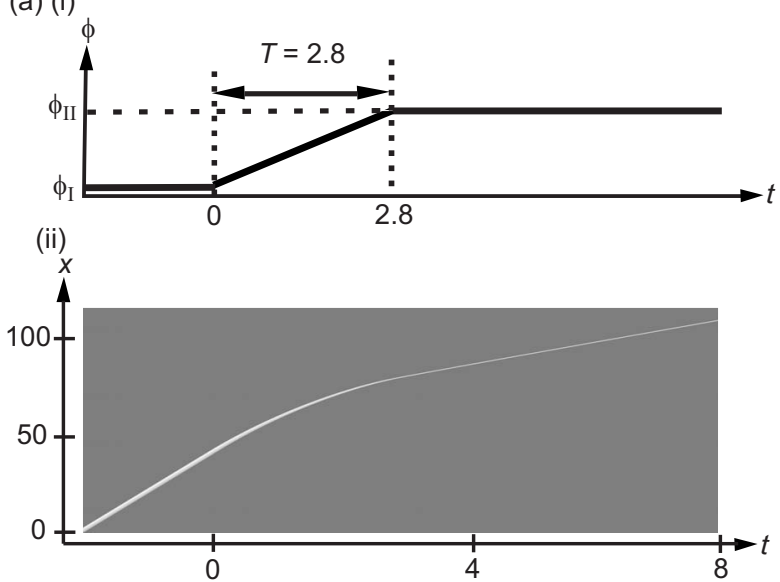

(b) (i)
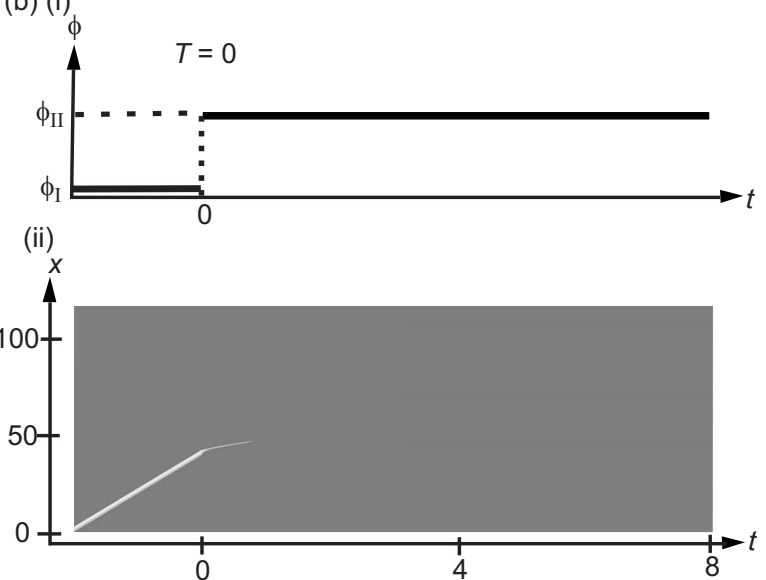

FIG. 3. Numerical results based on the Oregonator model, Eqs. (1)-(5). The parameter for light intensity was varied: (a) $T=2.8$ and (b) 0 . (i) Time trace of light intensity. We set $t=0$ at the moment that $\phi$ begins to change. (ii) Spatiotemporal plots of single-pulse propagation in one-dimensional excitable media for different $T$. The propagating pulse disappears in (b) and survives in (a).

$$
\begin{gathered}
\frac{\partial v}{\partial t}=D_{v} \nabla^{2} v+\frac{1}{\epsilon_{v}} g(u, v), \\
f(u, v, \phi)=u(1-u)-(f v+\phi) \frac{u-q}{u+q}, \\
g(u, v)=u-v,
\end{gathered}
$$

where $\phi$ represents an environmental factor, corresponding to the light intensity in our experiments. The variables $u$ and $v$ are the dimensionless concentrations of the activator and inhibitor, respectively. $D_{u}$ and $D_{v}$ are the diffusion coefficients of the activator and inhibitor. The functions $f(u, v, \phi)$ and $g(u, v)$ represent local reactions with characteristic time scales of $\epsilon_{u}$ and $\epsilon_{v}$. We introduce the temporal change in light intensity $\phi$ as follows:

$$
\phi(t)= \begin{cases}\phi_{I} \quad(t \leq 0) \\ \phi_{I}+\left(\phi_{I I}-\phi_{I}\right) t / T \quad(0 \leq t \leq T) \\ \phi_{I I} \quad(t \leq T) .\end{cases}
$$

We change the light intensity $\phi$ from $\phi_{I}=0.015$ to $\phi_{I I}$ $=0.0974$, just as in the experiment. $T$ is the duration of change in light intensity; i.e., the parameter $T$ is reciprocal to the rate of change in light intensity. It is not known whether there is a linear relationship between the light intensity in the real experiments and the parameter $\phi$ in the Oregonator model. Thus, in the numerical calculation, the parameter corresponding to the light intensity, $\phi$, is changed linearly for simplicity. How the manner of the change in light intensity, such as exponential, linear, etc., affects the pulse propagation is a matter for further investigation.

Throughout the calculations, we set $f=3.0, q=0.002, \epsilon_{u}$ $=0.004, \epsilon_{v}=1, D_{u}=1$, and $D_{v}=0.6$. Numerical calculations were carried out using the Euler method for the diffusion terms and the fourth-order Runge-Kutta method for the reac- tion terms. Neumann boundary conditions were adopted. The time step is taken to be $\Delta t=0.00002$, and the mesh size is taken to be $\Delta x=0.01$.

Figure 3 shows the numerical results; Fig. 3(a) and Fig. 3(b) are spatiotemporal plots of single-pulse propagation under slow $(T=2.8)$ and abrupt $(T=0)$ changes, respectively. When $T=2.8$, the traveling pulse survives with a change in light intensity [Fig. 3(a)]. On the other hand, when $T=0$, the pulse fails to propagate with a change in light intensity [Fig. 3(b)].

Figure 4 shows numerical results for the lifetime $\tau$ versus $T$. The lifetime $\tau$ is defined as the time interval from when the light intensity $\phi$ begins to change to when the traveling pulse disappears (the size of the traveling pulse in space units

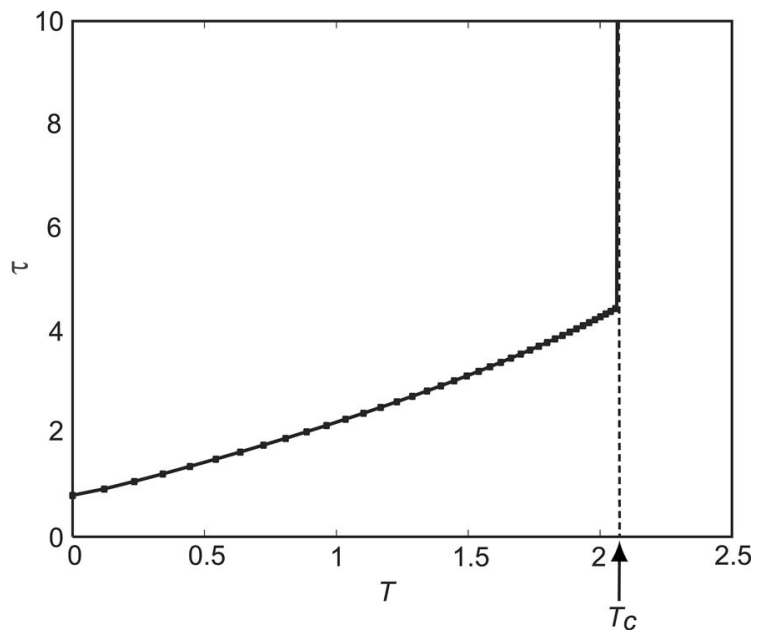

FIG. 4. Lifetime $\tau$ of the traveling pulse for different $T$ as deduced from the numerical calculation. A pulse fails to propagate when the duration of light growth $T$ is smaller than $T_{c}$, and survives when $T$ is larger than $T_{c}$. Calculations were performed with the modified Oregonator model [Eqs. (1)-(5)], where the parameters are $\phi_{I}=0.015, \phi_{I I}=0.0974, f=3, q=0.002, \epsilon_{u}=0.004, \epsilon_{v}=1, D_{u}=1$, and $D_{v}=0.6$. 
becomes 0 ). When $T>T_{c}, \tau$ diverges to infinity. Thus, a pulse survives under a slow change $\left(T>T_{c}\right)$, whereas it fails to propagate under an abrupt change $\left(T<T_{c}\right)$. These numerical results well reproduce the experimental trends.

\section{DISCUSSION}

The nature of pulse propagation in excitable media depends on the rate of change in an environmental parameter. The experimental and numerical results can be understood within the framework of reaction-diffusion systems. The FHN model $[17,18]$ has almost the same mathematical structure as the Oregonator model. We will discuss our results using the FHN model, which was modified similarly to the modified Oregonator model [Eqs. (3) and (4)], where the environmental parameter $\phi$ decreases the excitability of the system,

$$
\begin{gathered}
f(u, v, \phi)=u(1-u)(u-a)-v-\phi, \\
g(u, v)=u-\gamma v .
\end{gathered}
$$

Figure 5 shows the schematic difference between the responses to slow and abrupt inhibitory stimuli. Here, a crucial issue is what governs pulse propagation. Will the pulse propagate forward so that the new region excited by diffusion from the pulse front will grow, or will it disappear so that the excited region will shrink? To address this issue, the value of the excitation threshold $E_{\mathrm{th}}(v, \phi)$ in the vicinity of the traveling pulse $\left[E_{\mathrm{th}}^{f} \equiv E_{\mathrm{th}}\left(v_{f}, \phi\right)\right]$, which is defined in Fig. $5(\mathrm{c})$, is important $[22,23]$; here $v_{f}$ is the value of the slow variable $v(x, t)$ in the vicinity of the pulse front. It is well known that, when the excitation threshold $E_{\text {th }}(v, \phi)$ is very high, the newly excited region cannot expand, but rather can only shrink, if we nevertheless excite the medium [22].

Let us briefly explain that the value of $E_{\mathrm{th}}^{f}$ depends on the rate of change in the environmental parameter, and that under an abrupt change in $\phi$, the value of $E_{\mathrm{th}}^{f}$ is higher than that under a slow change. Since $u$ and $v$ are continuous with respect to $x, v_{f}$ is equivalent to $v(x, t)$ in the homogeneous area $[H$ in Fig. 5(a)] in front of the traveling pulse, where the diffusion terms are negligible $\left(\nabla^{2} u=\nabla^{2} v=0\right)$, and hence the dynamics of $u$ and $v$ can be described by the following equations:

$$
\begin{gathered}
\frac{d u_{h}}{d t}=\frac{1}{\epsilon_{u}} f\left(u_{h}, v_{h}, \phi(t)\right), \\
\frac{d v_{h}}{d t}=\frac{1}{\epsilon_{v}} g\left(u_{h}, v_{h}\right),
\end{gathered}
$$

where $u_{h}(t)$ and $v_{h}(t)$ are the values of $u$ and $v$ in this homogeneous area. Figure 5(b) shows the nullcline of the equations $\left[f_{I} \equiv f\left(u, v, \phi_{I}\right), f_{I I} \equiv f\left(u, v, \phi_{I I}\right)\right]$. With a change in the environmental parameter from $\phi=\phi_{I}$ to $\phi_{I I}$ the state in the homogeneous area $\left(u_{h}, v_{h}\right)$ changes from a stable fixed point under $\phi=\phi_{I}(P)$ to that under $\phi=\phi_{I I}(Q)$, where the trajectory from $P$ to $Q$ depends critically on the rate of change in the environmental parameter. Under a slow change, the state (a)

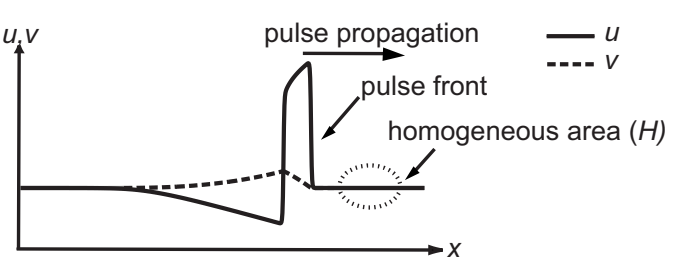

(b)

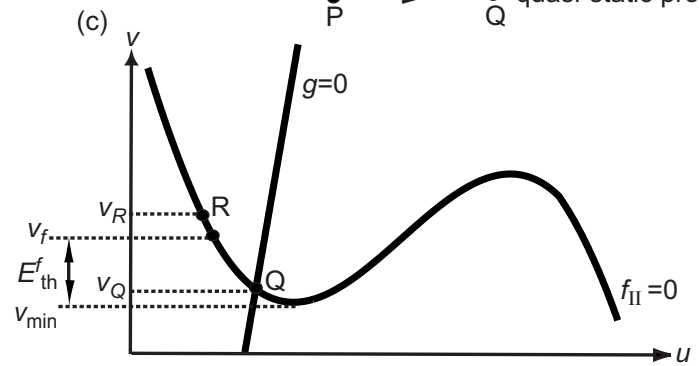

FIG. 5. Schematic representation of the response of the system to slow and abrupt changes in inhibitory stimuli. (a) Value of the slow variable in the vicinity of the pulse front $v_{f}$ can be considered to be equal to that in the homogeneous area $(H)$. (b) Nullclines of Eqs. (8) and (9) are shown for $\phi=\phi_{I}$ and $\phi_{I I}\left[f_{I}=f\left(u, v, \phi_{I}\right), f_{I I}\right.$ $\left.=f\left(u, v, \phi_{I I}\right)\right]$. The points $P$ and $Q$ give stable fixed points under $\phi=\phi_{I}$ and $\phi_{I I}$, respectively. Under a slow change, the resting state changes by a quasistatic process $(P Q)$. On the other hand, with an abrupt change, it changes by a two-step process $(P R Q)$. (c) Excitation threshold $E_{\mathrm{th}}(v, \phi)$, which can be estimated as $v-v_{\min }$ [22], where $v_{\min }$ is the value of $v$ at the bottom of the $u$ nullcline $f(u, v, \phi)=0$. Under a slow change in $\phi$, the value of the excitation threshold in the vicinity of the pulse front is equal to $E_{\mathrm{th}}\left(v_{Q}, \phi_{I I}\right)$. On the other hand, under an abrupt change, it changes from $E_{\mathrm{th}}\left(v_{R}, \phi_{I I}\right)$ to $E_{\mathrm{th}}\left(v_{Q}, \phi_{I I}\right)$, where $E_{\mathrm{th}}\left(v_{R}, \phi_{I I}\right)>E_{\mathrm{th}}\left(v_{Q}, \phi_{I I}\right)$.

changes in a quasistatic process $(P \rightarrow Q)$. On the other hand, under an abrupt change, the state changes in a two-step pro$\operatorname{cess}(P \rightarrow R \rightarrow Q)$, since $u$ and $v$ are fast and slow variables, respectively.

Thus, under a slow change, the value of $v_{f}$ at $\phi=\phi_{I I}$ is equal to $v_{Q}$. In contrast, under an abrupt change, it changes from $v_{R}$ to $v_{Q}\left(v_{R}>v_{Q}\right)$. Under an abrupt change in $\phi$, the value of the excitation threshold in the vicinity of the pulse front is higher than that under a slow change $\left[E_{\text {th }}^{f}\right.$ $=E_{\mathrm{th}}\left(v_{R}, \phi_{I I}\right)>E_{\mathrm{th}}\left(v_{Q}, \phi_{I I}\right)$ in Fig. 5(c)]. Hence, under an abrupt change, the new region excited by diffusion from the pulse front shrinks, resulting in disappearance of the traveling pulse.

To verify the correctness of our proposed mechanism, we performed a numerical calculation using the modified FHN model [Eqs. (1), (2), (6), and (7)]. Numerical calculations 
(a)

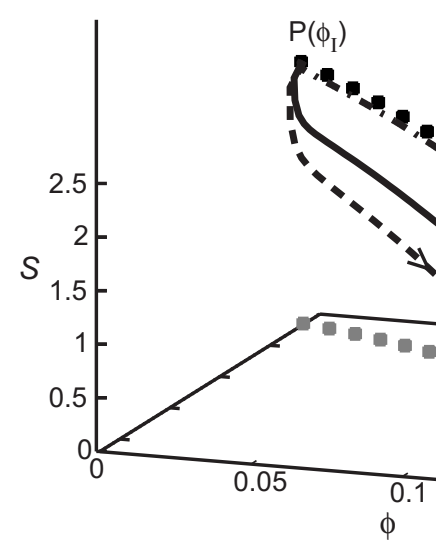

(b)

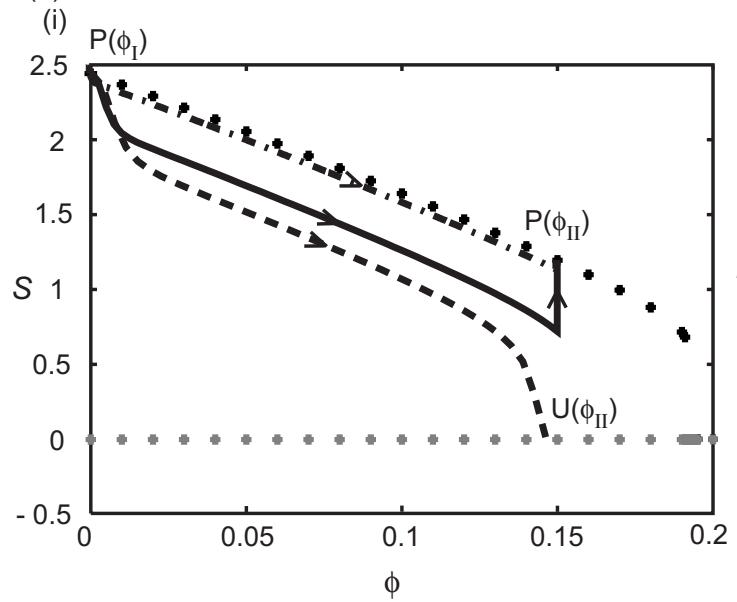

- stable pulse solution $P(\phi)$

- trivial uniform solution $\mathrm{U}(\phi)$

- - $T=4$

$T=6$

- ' - $T=40$

I 
and may possibly apply to the elegant procedures incorporated in information processing in biological systems.

\section{ACKNOWLEDGMENT}

We would like to thank Professor M. Nagayama (Kanazawa University, Japan), Professor S. Nakata (Nara University of Education, Japan), Dr. T. Ichino (Kinki University, Japan), Dr. K. Ueda (Kyoto University, Japan) and M.
Hörning (Kyoto University, Japan) for their helpful advice and discussions. This work was supported in part by the Japan Society for the Promotion of Science (JSPS) under a Grant-in-Aid for Creative Scientific Research to K.Y. (Grant No. 18GS0421), and by the Ministry of Education, Culture, Sports, Science and Technology of Japan (MEXT) under a Grant-in-Aid for Young Scientists (B) to H.K. (Grant No. 18740231).
[1] G. Bub, A. Shrier, and L. Glass, Phys. Rev. Lett. 88, 058101 (2002).

[2] A. O. Komendantov and N. I. Kononenko, J. Theor. Biol. 183, 219 (1996).

[3] M. A. Dahlem and S. C. Müller, Exp. Brain Res. 115, 319 (1997).

[4] E. Pálsson and E. C. Cox, Proc. Natl. Acad. Sci. U.S.A. 93, 1151 (1996).

[5] S. Jakubith, H. H. Rotermund, W. Engel, A. von Oertzen, and G. Ertl, Phys. Rev. Lett. 65, 3013 (1990).

[6] A. N. Zaikin and A. M. Zhabotinsky, Nature (London) 225, 535 (1970).

[7] A. T. Winfree, Science 175, 634 (1972).

[8] J. Keener and J. Sneyd, Mathematical Physiology (Springer, New York, 1998).

[9] L. Kuhnert, Naturwiss. 73, 96 (1986).

[10] L. Kuhnert, Nature (London) 319, 393 (1986).

[11] K. Agladze, V. Voignier, E. Hamm, F. Plaza, and V. Krinsky, J. Phys. Chem. 100, 18764 (1996).

[12] S. Kádár, T. Amemiya, and K. Showalter, J. Phys. Chem. A
101, 8200 (1997).

[13] K. Agladze, Á. Tóth, T. Ichino, and K. Yoshikawa, J. Phys. Chem. A 104, 6677 (2000).

[14] T. Sakurai, E. Mihaliuk, F. Chirila, and K. Showalter, Science 296, 2009 (2002).

[15] R. J. Field and R. M. Noyes, J. Phys. Chem. 60, 1877 (1974).

[16] J. J. Tyson and P. C. Fife, J. Phys. Chem. 73, 2224 (1980).

[17] R. Fitzhugh, Biophys. J. 1, 445 (1961).

[18] J. Nagumo, S. Arimoto, and S. Yoshizawa, Proc. IRE 50, 2061 (1962).

[19] J. Gorecki, J. N. Gorecka, K. Yoshikawa, Y. Igarashi, and H. Nagahara, Phys. Rev. E 72, 046201 (2005).

[20] H. J. Krug, L. Pohlmann, and L. Kuhnert, J. Phys. Chem. 94, 4862 (1990).

[21] T. Amemiya, S. Kádár, P. Kettunen, and K. Showalter, Phys. Rev. Lett. 77, 3244 (1996).

[22] V. Krinsky and A. Pumir, Chaos 8, 188 (1998).

[23] J. J. Tyson and J. P. Keener, Physica D 32, 327 (1988).

[24] H. P. McKean, Adv. Math. 4, 209 (1970). 